\title{
ON FIXED POINTS OF AUTOMORPHISMS OF FINITELY GENERATED FREE GROUPS
}

\author{
BY S. M. GERSTEN
}

\begin{abstract}
If $\phi$ is an automorphism of a finitely generated free group, then $\operatorname{Fix}(\phi)$ is finitely generated.
\end{abstract}

0. Introduction. A conjecture attributed to G. P. Scott [St2] states that the fixed points of an automorphism $\phi$ of a finitely generated (fg) free group $F$ is fg. It is known that this is the case if $\phi$ is periodic $[\mathbf{D}-\mathbf{S}]$ or geometric $[\mathbf{J}-\mathbf{S}]$, where $\phi$ is called geometric if it is the induced map on $\pi_{1}$ of a homeomorphism of $(M, x)$, where $M$ is a compact bounded two-dimensional manifold and $x \in$ $M$. In a recent article [G2] we showed that Scott's conjecture is valid for automorphisms of $\pi_{1}$ of a finite graph induced by change of maximal trees.

In this article we announce the affirmative solution of Scott's conjecture. The methods involved are an extension of methods of [G2] and blend combinatorics, topology, and group theory.

1. Graphs. We work with a combinatorial notion of a graph introduced in [G1] (and based on an idea of Serre's [S]). A graph $X$ is a nonempty set with involution $x \rightarrow \bar{x}$ (so $\overline{\bar{x}}=x$ ) and retraction $i: X \rightarrow V(X)$, where $V(X)$ is the fixed point set of $x \rightarrow \bar{x}$. Thus $i(i(x))=i(x)$. One defines $\tau(x)=: i(\bar{x})$. Intuitively, $V(X)$ is the set of vertices, $X-V(X)=: E(X)$ is the set of edges, $i$ is the initial vertex and $\tau$ is the terminal vertex.

A morphism $f: X \rightarrow X^{\prime}$ of graphs is a function such that $f(\bar{x})=\overline{f(x)}$, and $i(f(x))=f(i(x)), x \in X$. The category of graphs has a final object, fibre products and push outs. In addition, there is a geometrical realization functor $B X$ which assigns to $X$ a 1-dimensional CW complex. This permits us to use the geometrical language of maximal trees, fundamental group and homotopy equivalence, although the notions have purely combinatorial definitions in the category of graphs.

A morphism $f: X \rightarrow Y$ is called an immersion [St1] if $f_{v}: \operatorname{Star}_{v}(X) \rightarrow$ $\operatorname{Star}_{f(v)}(Y)$ is injective for each $v \in V(X)$. Here $\operatorname{Star}_{v}(X)=\{e \in E(X) \mid i e=$ $v\}$. Immersions preserve reduced paths and hence induce injective maps on $\pi_{1}$ [St1]. Of crucial importance in our work is the degenerate set $D f$ of a morphism $f: X \rightarrow Y$; here $D f=:\{x \in X \mid f(x) \in V(Y)\}$.

If $v \in V(X)$ and $f: X \rightarrow Y$ is a morphism, denote by $f_{*}$ the induced map $\pi_{1}(X, v) \rightarrow \pi_{1}(Y, f(v))$.

Received by the editors November 24, 1982 and, in revised form, January 26, 1983.

1980 Mathematics Subject Classification. Primary 20F34, 94C15, 57M05, 57M15, 05C10, $05 \mathrm{C} 25$.

Key words and phrases. Free group, automorphism, fixed point, graph, fundamental group. 
1.1. ThEOREM. If $\phi \in \operatorname{Aut}\left(\pi_{1}(Y)\right)$ where $Y$ is a finite graph with one vertex, there exists a finite graph $X, v \in V(X)$, and morphisms $f, f^{\prime}: X \rightarrow Y$ s.t.

(1) $f_{*}$ and $f_{*}^{\prime}$ are surjective with the same kernel,

(2) $f_{*}^{\prime} \circ f_{*}^{-1}=\phi$, and

(3) $D f$ and $D f^{\prime}$ contain maximal trees of $X$.

The proof makes use of Whitehead's algorithm [L-S] for decomposing $\phi$ as a product of Whitehead automorphisms, and a theorem of ours, [G2, 5.5], representing a Whitehead automorphism as a change-of-maximal-tree automorphism (blow-up followed by blow-down in the aeolean terminology of [G1]).

After modifying $X, f, f^{\prime}$ in 1.1 by edge collapses, edge folds [St1] and by removing extraneous edges, one proves

1.2. THEOREM. In the notation of 1.1 , one may assume $f, f^{\prime}$ satisfy

G1. $D f, D f^{\prime}$ are maximal trees of $X$,

G2. $f \mid(X-E(D f))$ is an immersion and $f^{\prime} \mid\left(X-E\left(D f^{\prime}\right)\right)$ is an immersion, and

G3. $f_{*}$ and $f_{*}^{\prime}$ are surjective with the same kernel.

In addition, G1, G2, and G3 imply then

G4 ("path surgery"). If $x, y \in E(X)-E(D f)$ are such that $f(x)=f(y)$,

$$
l\left(\bar{x} \cdot[i x, i y]_{D f} \cdot y\right)>l\left([\tau x, \tau y]_{D f}\right)
$$

and similarly for $f^{\prime}$ replacing $f$. Here $[v, w]_{D f}$ is the geodesic in the maximal tree $D f$ from vertex $v$ to vertex $w$, and "l" denotes the length of a path.

Let $\mathcal{E}_{v}=\left\{u \in \pi_{1}(X, v) \mid f_{*}(u)=f_{*}^{\prime}(u)\right\}$. It is easily seen that $f_{*}\left(\mathcal{E}_{v}\right) \cong$ $\operatorname{Fix}(\phi)=\left\{\eta \in \pi_{1}(Y) \mid \phi(\eta)=\eta\right\}$. With these preliminaries we can state our main result.

1.3. TheOREm. Assume $\phi \in \operatorname{Aut}\left(\pi_{1}(Y)\right)$, where $Y$ is a finite graph with one vertex. Assume $f, f^{\prime}: X \rightarrow Y, v \in V(X)$ satisfy G1-G4 in 1.2, with $f_{*}^{\prime} \circ f_{*}^{-1}=$ $\phi$. Then $f_{*}\left(\varepsilon_{v}\right) \cong \operatorname{Fix}(\phi)$ is finitely generated.

We remark that in all examples we have studied, as well as in the results of [D-S], [J-S], and [G2], $\operatorname{rank}(\operatorname{Fix} \phi) \leq h_{1}(Y)$ (in the situation of [G2] $X \simeq Y$ ). We do not know if this inequality holds in general.

We shall outline the proof of 1.3 in the remainder of this article. Properties G1-G4 of $f, f^{\prime}: X \rightarrow Y$ will be assumed throughout without further mention.

\section{Representation theorems.}

2.1. A reduced path $\left(x_{1}, \ldots, x_{n}\right)$ in $X$ [St1] is called $f$ reduced if, after deleting all $x_{i} \in D f$ and applying $f$ to the remaining edges, the resulting path in $Y$ is reduced. The path $p$ in $X$ is called $\left(f, f^{\prime}\right)$ reduced if it is both $f$ - and $f^{\prime}$ reduced. A path $p$ in $X$ is called invariant if $f_{*} p=f_{*}^{\prime} p$, where $f_{*} p$ denotes the reduced path in $Y$ in the homotopy class of $f(p)$. An $\left(f, f^{\prime}\right)$ reduced invariant path $p$ in $X$ is called minimal if no nontrivial initial segment of $p$ is invariant. We denote by $\mathcal{M}$ the set of minimal $\left(f, f^{\prime}\right)$ reduced invariant paths.

2.2. THEOREM. If $\eta \in f_{*}\left(\mathcal{E}_{v}\right)$, then there are only finitely many $\left(f, f^{\prime}\right)$ reduced paths $w$ in $X$ with $f_{*} w=f_{*}^{\prime} w=\eta$; and they are determined by $a$ simple algorithm. 
This algorithm is based on the algorithm of [G2, §4].

2.3. THEOREM. For each path $w$ in $X \exists$ an $\left(f, f^{\prime}\right)$ reduced path $w_{1}$ s.t.

(1) $i w=i w_{1}, \tau w=\tau w_{1}$, and

(2) $f_{*} w=f_{*} w_{1}, f_{*}^{\prime} w=f_{*}^{\prime} w_{1}$.

The proof is accomplished by doing path-surgeries. The process converges by $\mathrm{G} 4$.

2.4. CoROllaRY. If $\eta \in f_{*}\left(\mathcal{E}_{v}\right), \exists$ an $\left(f, f^{\prime}\right)$ reduced circuit $w$ based at $v$ s.t. $w$ is invariant and $f_{*} w=\eta$.

Writing $w$ in 2.4 as a path product of paths in $\mathcal{M}$, one deduces

2.5. Corollary. $f_{*}\left(\varepsilon_{v}\right)=f_{*}\left(\langle\mathcal{M}\rangle \cap \pi_{1}(X, v)\right)$.

Here $X$ is immersed in $Z=: X /[V(X)=p t]$ and the intersection on the right-hand side takes place in $\pi_{1}(Z)$.

\section{Uniqueness theorems.}

3.1. Definition. If $e \in E(X)$, let $\mathcal{M}_{e}$ denote the set of paths $p \in \mathcal{M}$ with initial edge $e$. A path of minimal length in $\mathcal{M}_{e}$ is called standard. If $p$ and $p^{\prime}$ are paths in $X$ with $i p=i p^{\prime}, \tau p=\tau p^{\prime}, f_{*}(p)=f_{*}\left(p^{\prime}\right)$ and (of necessity, by G3) $f_{*}^{\prime}(p)=f_{*}^{\prime}\left(p^{\prime}\right)$, we write $p \stackrel{\circ}{=} p^{\prime}$.

3.2. THEOREM. If $p$ and $p^{\prime}$ are in $\mathcal{M}_{e}$ and if $p$ is standard, then $f_{*}(p)$ is an initial segment of $f_{*}\left(p^{\prime}\right)$.

The proof is accomplished by careful examination of an algorithm which constructs all elements of $\mathcal{M}$.

3.3. COROLLARY. If $p$ and $p^{\prime}$ are in $\mathcal{M}_{e}$ and are both standard, then $p \stackrel{\circ}{=} p^{\prime}$.

\section{Main result.}

4.1. LEMMA. Let $w, w^{\prime}$ be invariant paths in $X$ such that $i(w)=i\left(w^{\prime}\right)$ and $f_{*}(w)=f_{*}\left(w^{\prime}\right)$. Then $\tau(w)=\tau\left(w^{\prime}\right)$.

We now reduce the (possibly infinite) set $\mathcal{M}$. For each $e \in E(X)$ such that $\mathcal{M}_{e} \neq \varnothing$, pick one standard path $w_{e}$. Let $\mathcal{M}^{\prime}$ be the set of $w_{e}$ chosen.

4.2. THEOREM. $f_{*}\left(\left\langle\mathcal{M}^{\prime}\right\rangle \cap \pi_{1}(X, v)\right)=f_{*}\left(\langle\mathcal{M}\rangle \cap \pi_{1}(X, v)\right)$.

One shows by induction on $l\left(f_{*}(w)\right)$ that if $w \in \mathcal{M}$, then $w \stackrel{\circ}{=} w^{\prime}$, where $w^{\prime}$ is a path product of paths in $\mathcal{M}^{\prime}$.

ProOF OF THEOREM 1.3. By 2.5 and 4.2 it suffices to prove that $\left\langle\mathcal{M}^{\prime}\right\rangle \cap$ $\pi_{1}(X, v)$ is finitely generated. But $\mathcal{M}^{\prime}$ is a finite set, so the result follows from a theorem of Howson's [St1, 5.6].

One can deduce from $H$. Neumann's inequality, $[\mathbf{G 1}, 4.1]$, the inequality rank $(\operatorname{Fix}(\phi))-1 \leq 2(\# E(X)-1) \cdot\left(h_{1}(X)-1\right)$, where $h_{1}(X)$ is the first betti number of $X$, provided $\operatorname{Fix}(\phi) \neq\{1\}$.

\section{Automorphisms with few fixed points.}

5.1. TheOREM. ("Antisymmetry principle"). Suppose $w \in \mathcal{M}$ has initial edge in $D f$. Then $w$ must have its terminal edge in $D f^{\prime}$. 
5.2. Proposition. Suppose $e \in E(D f)$ is s.t. if $f^{\prime}(e)=f(x)$ for any $x \in E(X)$, then $[\tau e, i x]_{D f}$ has initial edge $\bar{e}$. Then $\mathcal{M}_{e}=\varnothing$.

From 5.1 and 5.2, together with 2.5, we can produce examples of automorphisms with no nontrivial fixed points. In particular all the examples of [Sq] can be treated by our methods. This is of interest in accumulating evidence for Stallings' conjecture [St2], that a PV automorphism $\phi$ of a $f g$ free group of rank $\geq 3$ has $\operatorname{Fix}(\phi)=\{1\}$.

We should like to express our gratitude to John Stallings, who saw us through some very difficult years. We learned of the Scott conjecture from him. He offered us continual encouragement as we came to understand the problem.

\section{BIBLIOGRAPHY}

[G1] S. M. Gersten, Intersections of finitely generated subgroups of free groups and resolutions of graphs, Invent Math. (to appear).

[G2] _ On fixed points of certain automorphisms of free groups, preprint.

[St1] J. R. Stallings, The topology of finite graphs, Invent Math. (to appear).

[St2] _ Topologically unrealizable automorphisms of free groups, Proc. Amer. Math. Soc. 84 (1982), 21-24.

[D-S] J. L. Dyer and G. P. Scott, Periodic automorphisms of free groups, Comm. Algebra 3 (1975), 195-201.

[J-S] W. Jaco and P. B. Shalen, Surface homeomorphisms and periodicity, Topology 16 (1977), 347-367.

[S] J.-P. Serre, Arbres, analgames, $S l_{2}$, Astérisque, no. 46, Société Mathématique de France, Paris, 1977.

[Sq] C. Squier, Fixed points and finite orbits of free group automorphisms, preprint.

[L-S] R. C. Lyndon and P. E. Schupp, Combinatorial group theory, Ergebnisse der Mathematik und ihrer Grenzgebiete, Band 89, Springer-Verlag, Berlin and New York, 1977.

DEPARTMENT OF MATHEMATICS, UNIVERSity of UTAH, SALT LAKE City, UTAH 84112 